\title{
Substituição de Artemia sp. pelo Rotífero Brachionus plicatilis na Larvicultura do Camarão-d'Água-Doce (Macrobrachium rosenbergii De Man, 1879) ${ }^{1}$
}

\author{
Luciana Almada Thomaz ${ }^{2}$, Lídia Miyako Yoshii Oshiro ${ }^{3}$, Andrea Cecchetto Bambozzi ${ }^{4}$, \\ José Teixeira de Seixas Filho ${ }^{5}$, Leonardo Azevedo da Silva Rosadas ${ }^{6}$
}

RESUMO - Conduziu-se o presente trabalho com o objetivo de avaliar o efeito da substituição dos náuplios de Artemia sp. pelo rotífero enriquecido e congelado (Brachionus plicatilis, O.F. Müller, 1786) sobre a sobrevivência e peso seco das larvas. O experimento foi conduzido em blocos casualizados, sendo testados quatro regimes alimentares na larvicultura do camarão M. rosenbergii, com quatro repetições por tratamento. Nos regimes alimentares, realizaram-se substituições progressivas de náuplios de Artemia sp. (nas) pelo rotífero Brachionus plicatilis (rots) e testaram-se os seguintes tratamentos: $100 \%$ B. plicatilis (30 rots/mL) (T1); 100\% Artemia sp. (5 nas/mL) (T2), 60\% Artemia sp. (3 nas $/ \mathrm{mL})+40 \%$ B. plicatilis (12 rots/mL) (T3) e 40\% Artemia sp. (2 nas $/ \mathrm{mL})+60 \%$ B. plicatilis (18 rots/mL) (T4), adicionando-se a estes tratamentos ração úmida. Os resultados da sobrevivência final em pós-larvas foram analisados pelo teste do $\mathrm{X}^{2}$ e demonstraram não haver diferenças significativas do tratamento T2 $(68,36 \%)$ sobre os tratamentos T3 $(68,76 \%)$ e T4 $(64,60 \%)$. O tratamento T1 (100\% B. plicatilis) apresentou mortalidade total no 14 o dia do experimento. O peso seco médio das pós-larvas foi analisado estatisticamente pela ANOVA, não apresentando diferenças significativas entre os valores de 3,29 mg (T2), 3,08 mg (T3) e 3,38 mg (T4). A taxa de mortalidade baseada no número de larvas encontradas mortas diariamente, em relação ao número de larvas estocadas inicialmente, foi maior para todos os tratamentos nos primeiros 15 dias do experimento. Portanto, os resultados demonstraram que a substituição total de rotíferos enriquecidos e congelados acarretou a mortalidade total das larvas, mas a substituição parcial de $40 \%$ e $60 \%$ dos náuplios de Artemia sp. pelo B. plicatilis foi viável, não apresentando prejuízos significativos em termos de sobrevivência e peso seco das pós-larvas.

Palavras-chave: alimentação, aqüicultura, camarão-d'água-doce, larvicultura, Macrobrachium rosenbergii, rotífero

\section{Replacement of the Artemia sp. for the Rotifer Brachionus plicatilis in the Hatchering of the Freshwater Prawn (Macrobrachium rosenbergii De Man, 1879)}

ABSTRACT - This work was carried out to identify the replacement of the nauplii Artemia sp. for the enriched and frozen rotifer (Brachionus plicatilis O.F. Müller, 1786) on the larval development and survival of the freshwater prawn M. rosenbergii. The experiment was developed according to the following treatments: $100 \%$ B. plicatilis (30 rots/ mL) (T1); 100\% Artemia sp. (5 nas/ $\mathrm{mL}$ ) (T2), 60\% Artemia sp. (3 nas/ mL) + 40\% B. plicatilis (12 rots/ mL) (T3) e 40\% Artemia sp. (2 nas/ mL) + 60\% B. plicatilis (18 rots/ mL) (T4). Humid ration was added to all treatments. The results of the final survival in post-larvae were analyzed by the $\mathrm{X}^{2}$ test and demonstrated no significant differences from treatment T2 (68.36\%) to T3 and T4: 68.76\% and 64.60\%, respectively. T1 treatment $\left(100 \%\right.$ B. plicatilis) presented total mortality at $14^{\text {th }}$ day of the experiment. Average dry weight of the pos-larvae was analyzed statistically by ANOVA and showed no significant differences in values: $3.29 \mathrm{mg}$ (T2), $3.08 \mathrm{mg}$ (T3) and $3.38 \mathrm{mg}$ (T4). No significant differences among treatments T2, T3 and T4 were observed. The best mortality rate was observed from 1 to 15 day of hatchery based on the number of dead larval stored in the daily material siphoned. Therefore, these results suggest that the total substitution of the enriched and frozen rotifer is not recommended due to a total mortality of larvae, whereas the partial replacement of $40 \%$ and $60 \%$ of the Artemia sp nauplii by the enriched and frozen rotifer is possible, with no significant damages to survival and post-larval growth.

Key Words: aquaculture, feeding, freshwater prawn, hatchery, Macrobrachium rosenbergii, rotifer

\footnotetext{
${ }^{1}$ Parte da Dissertação de Mestrado em Zootecnia apresentada pelo primeiro autor a UFRRJ.

${ }^{2}$ Eng. Agrônoma, aluna do Programa de Pós-graduação em Zootecnia do Instituto de Zootecnia UFRRJ (almadalt@yahoo.com.br).

3 Professora Adjunta 4 - Departamento de Produção Animal. Instituto de Zootecnia da UFRRJ (oshiro@ufrrj.br)

${ }^{4}$ Zootecnista - aluna do Programa de Pós-graduação em Zootecnia do Instituto de Zootecnia UFRRJ (abambozzi@yahoo.com.br).

${ }^{5}$ Biólogo, DS em Zootecnia, Pesq. FIPERJ (joseseixas@fiperj.rj.gov.br)

${ }^{6}$ Aluno de graduação em Ciências Biológicas UFRRJ (leo-rosadas@hotmail.com).
} 


\section{Introdução}

A criação do $M$. rosenbergii (De Man, 1879) envolve a fase de larvicultura, em que a obtenção de pós-larvas é um dos principais obstáculos ao desenvolvimento de cultivos comerciais de camarõesd'água-doce. Trata-se de uma atividade dependente da produção de alimentos vivos e que requer cuidados e monitoramento freqüente, tornando-se um dos fatores mais importantes no desenvolvimento larval (Yufera et al., 1985; Freeman, 1990).

A administração de uma alimentação adequada é fundamental para a larvicultura, pois afeta diretamente a sobrevivência e o tempo de desenvolvimento larval (New et al. 2000; Cestarolli et al., 1997). É importante que mais de um alimento de mesma natureza seja utilizado para que o nível de aminoácidos, vitaminas e demais nutrientes requeridos seja o mais completo possível (Valenti, 1991; Barros \& Valenti, 2003). Portanto, uma dieta alimentar é considerada ótima quando tem seus teroes de proteínas, de lipídios e de carboidratos balanceados (Whyte et al., 1989, 1994).

As técnicas existentes para o desenvolvimento larval são predominantemente dependentes de alimentos vivos, que requerem cuidados especiais e monitoramento freqüente, tornando-se muito dispendiosas. Várias fontes de alimento animal (vivo ou congelado) têm sido experimentadas. Entretanto, a mais comum hoje é o náuplio de Artemia sp., graças à praticidade de seu uso e seu alto valor nutritivo (Sorgeloos et al., 1983).

Na aqüicultura, o uso de Artemia sp. tem causado alguns transtornos, pois a reduzida disponibilidade de cistos, em função da limitação de locais para sua produção e processamento (Sorgeloos, 1980) contribuem para queda em sua produção, tornando os preços elevados, conforme demanda (Lavens et al., 2000). Além disso, há considerável variação na qualidade dos cistos quanto à taxa de eclosão e ao valor nutricional do náuplio eclodido (Sorgeloos \& Léger, 1992).

Vários organismos aquáticos foram estudados no intuito de se obter novas fontes protéicas e reduzir os custos de produção das larvas de camarão. Entre os animais utilizados na tentativa de substituição total ou parcial dos náuplios de Artemia sp., foram experimentadas algas (Alam et al., 1991, 1993a, 1993b), o cladócero Moina spp. (Aniello \& Singh, 1980), o nematóide Panagrellos redivivus, (Cerqueira, 1989; Silva \& Rodrigues, 1997) e o rotífero Brachionus plicatilis (Seixas Filho et al., 1984, 1985, 2000; Lovett
\& Felder, 1988), mas os resultados não foram satisfatórios quando comparados aos obtidos com Artemia sp. (New et al., 2000; Correia et al., 2000).

Para a seleção desses organismos vivos, são considerados o tamanho adequado, o valor nutritivo compatível e a facilidade de cultivo em grande escala (Lavens et al., 2000; Barros \& Valenti, 2003). O rotífero B. plicatilis satisfaz estes requerimentos e tem sido usado como alimento animal oferecido a peixes tropicais e larvas de camarões (Seixas Filho et al., 1984, 1985 e 2000).

No entanto, não tem sido possível a substituição completa de Artemia sp. na larvicultura de M. rosenbergii. Este fato pode ser atribuído ao limitado conhecimento das necessidades nutricionais das larvas e às dietas disponíveis, nutricionalmente inadequadas (Lovett \& Felder 1988; Kissil \& Koven, 1990; Van Der Meeren, 1991; Sorgelloos \& Léger, 1992; Stottrup \& Attramadal, 1992; Silva \& Rodrigues, 1997).

Este trabalho foi conduzido com o objetivo de avaliar o efeito da substituição total ou parcial dos náuplios de Artemia sp. pelo rotífero B. plicatilis enriquecido e congelado na alimentação de larvas do camarão $M$. rosenbergii e analisar seu desempenho a partir da sobrevivência e do peso seco das póslarvas ao final da larvicultura.

\section{Material e Métodos}

O experimento foi realizado no Laboratório da Estação de Aqüicultura Almirante Paulo Moreira (EAAPM) da Fundação do Instituto de Pesca do Estado do Rio de Janeiro (FIPERJ). O experimento foi realizado no período de 18 de agosto de 2000 a abril de 2001, utilizando-se larvas de camarões-d'água-doce (M. rosenbergii) eclodidas de várias fêmeas ovígeras, oriundas da Fazenda Santa Helena, Silva Jardim (RJ).

As larvas do camarão-d'água-doce ( $M$. rosenbergii) foram estocadas em densidade de, aproximadamente, de 100 larvas. $^{-1}$, em 16 tanques retangulares, com capacidade individual de 33 litros, revestidos em seu interior com tinta epóxi preta, para impedir a fixação de agentes contaminantes e facilitar a visualização e apreensão do alimento (náuplios de Artemia sp., rotífero enriquecido e partículas de ração) pelas larvas de camarão. Os aspectos físicoquímicos da água dos tanques de larvicultura do camarão-d'água-doce foram monitorados durante todo o período experimental. O valor do $\mathrm{pH}$ apresentou variação média de 8,0 a 8,3. Os níveis de amônia

R. Bras. Zootec., v.33, n.6, p.1928-1933, 2004 (Supl. 2) 
mantiveram-se inferiores a 0,1 ppm e o oxigênio manteve-se sempre próximo à saturação $(7,5-8,0$ ppm). A temperatura manteve-se em $28^{\circ} \mathrm{C}\left( \pm 1^{\circ} \mathrm{C}\right)$, com auxílio de aquecedores acoplados a termostato, e a salinidade foi mantida a $16 \mathrm{ppm}$. Portanto, os valores destes parâmetros foram mantidos dentro da faixa considerada adequada para o bom desempenho dos animais, de acordo com vários autores (New, 1976, 1990, 1995; Uno \& Soo, 1969; Valenti, 1991, 1998; Seixas Filho et al., 1984, 1985, 2000).

Desde o primeiro dia de criação, foi fornecido às larvas alimento inerte (ração úmida), administrado ad libitum de forma equivalente para todos os tratamentos. A ração foi fornecida três vezes ao dia, às 8 , 10 e $12 \mathrm{~h}$, enquanto o alimento vivo foi fornecido às 17 h, após a limpeza dos tanques.

Cada unidade experimental recebeu aeração constante, por meio de soprador elétrico e mangueiras plásticas 3/16” providas de pedras porosas em suas extremidades. A aeração serviu também para manter as partículas alimentares em suspensão, proporcionando distribuição homogênea das larvas por toda coluna d'água, evitando o aumento da taxa de canibalismo.

A limpeza dos tanques foi feita diariamente às $13 \mathrm{~h}$, por sifonamento, retirando-se a aeração e aguardando a decantação das partículas. Em seguida, por intermédio de uma mangueira plástica transparente de 3/4”, foram sifonadas as sobras de alimento, de matéria orgânica e de fezes das larvas concentradas no fundo do tanque, em baldes de fibra de vidro providos de tela de $150 \mu \mathrm{m}$ para retenção das larvas que se encontravam próximas ao fundo. Efetuada esta operação, recolocavase a aeração, renovava-se $90 \%$ do volume da água dos tanques e devolvia ao sistema as larvas eventualmente sifonadas. Diariamente, foi feita a contagem das larvas mortas encontradas no material sifonado.

Ao final do experimento, quando $90 \%$ das larvas completaram a metamorfose e passaram à fase de pós-larva, a salinidade da água foi gradativamente reduzida a zero e o experimento foi encerrado, obtendo-se assim, os dados do período de larvicultura.

A sobrevivência durante a larvicultura foi analisada por meio de curvas de sobrevivência da população em cada tratamento, sendo estimada pela subtração da população inicial, pelo número de larvas encontradas mortas diariamente nos resíduos do tanque de larvicultura.

Para analisar o peso seco das pós-larvas, foram separadas ao acaso 20 pós-larvas de cada unidade experimental (tanque), totalizando 80 pós-larvas por bloco, que foram acondicionadas por 48 horas em estufa ventilada a $65^{\circ} \mathrm{C}$.

O experimento foi conduzido em blocos ao acaso, onde foram testados quatro regimes alimentares na larvicultura em circuito aberto, com quatro repetições por tratamento. Nos regimes alimentares, foram feitas substituições progressivas do náuplio de Artemia sp. (nas) pelo rotífero B. plicatilis (rots) e foram testados os seguintes tratamentos: $100 \%$ B. plicatilis (30 rots/ mL) (T1); 100\% Artemia sp. (5 nas/ mL) (T2), 60\% Artemia sp., (3 nas/ mL) + $40 \%$ B. plicatilis (12 rots/ mL) (T3) e $40 \%$ Artemia sp., (2 nas/ mL) $+60 \%$ B. plicatilis (18 rots/ mL) (T4). Os valores de substituição utilizados foram baseados nos estudos de Seixas Filho et al. (1984), que compararam o valor nutricional do náuplio de Artemia sp. e do rotífero B. plicatilis e constataram que seriam necessários 30 rotíferos para cada cinco náuplios de artemia, para efeito de equivalência.

A análise estatística do peso seco das pós-larvas foi realizada por análise de variância, a 5\% de significância, no programa SAEG 5.0 (Sistema de Análises Estatísticas e Genéticas) (Euclydes, 1982).

O modelo estatístico utilizado para análise dos dados de peso seco médio foi:

$$
\mathrm{Y}_{\mathrm{ij}}=\mathrm{m}+\mathrm{T}_{\mathrm{i}}+\mathrm{B}_{\mathrm{j}}+\mathrm{E}_{\mathrm{ij}}
$$

em que: $Y_{i j}=$ observação relativa ao tratamento i no bloco j; $\mathrm{m}=$ efeito médio geral; $\mathrm{T}_{\mathrm{i}}=$ efeito do tratamento i, i= 100\% rotífero; $100 \%$ Artemia; $60 \%$ Artemia + 40\% rotífero; $40 \%$ Artemia $+60 \%$ Rotífero; $\mathrm{B}_{\mathrm{j}}=$ efeito do bloco $\mathrm{j}$; $\mathrm{E}_{\mathrm{ij}}=$ erro aleatório associado ao tratamento i no bloco $\mathrm{j}$.

Para a análise do resultado de sobrevivência, foi utilizado o método não-paramétrico do qui-quadrado.

\section{Resultados e Discussão}

A sobrevivência das pós-larvas após a metamorfose total (35 dias) não mostrou diferença significativa $(\mathrm{P}>0,05)$ quando realizada a análise estatística pelo qui-quadrado entre os animais submetidos aos tratamentos, T2, T3 e T4 (Tabela 1).

As curvas de sobrevivência até o $5^{\circ}$ dia, para todos os tratamentos, foram muito semelhantes, mas, posteriormente, verificou-se uma queda na sobrevivência do tratamento $\mathrm{T} 1$ (100\% B. plicatilis), que, ao $14^{\circ}$ dia, chegou à mortalidade total da população. Isto pode ser um indicativo de que os rotíferos enriquecidos e congelados não foram suficientes em termos nutricionais na dieta dessas larvas (Figura 1). 
Silva \& Rodrigues (1997) também encontraram nos primeiros dez dias de larvicultura, a mortalidade total de larvas de $M$. rosenbergii, quando alimentadas somente com nematóides (Panagrellus redivivus). Porém, Seixas Filho et al. (1984) e Lovett $\&$ Felder (1988) verificaram baixa taxa de sobrevivência das pós-larvas de $M$. rosenbergii $(0,6 \%$ e $0,8 \%$ ), quando utilizaram os rotíferos sem enriquecimento nas densidades de 30 e 50 rots/mL, respectivamente, na substituição total dos náuplios de Artemia sp.

As curvas de sobrevivência das larvas que receberam os tratamentos T2 (100\% Artemia sp.), T3 (60\% Artemia sp. $+40 \%$ B. plicatilis) e T4 $(40 \%$ Artemia sp. $+60 \%$ B. plicatilis) foram muito semelhantes, ocorrendo mortalidade mais acentuada nos primeiros dez dias e estabilização a partir do $20^{\circ}$ dia (Figura 1).

Os resultados positivos com os animais, como a sobrevivência em pós-larvas e os cromatóforos das larvas com coloração intensa, caracterizando bom estado nutricional, sugerem que o enriquecimento nutricional de rotíferos utilizando solução de fermento biológico (Saccharomyces cerevisae) e a microalga (Nanocloropsi oculata) + emulsão de óleo de fígado de bacalhau (preparo comercial) por 12 horas sobre o meio de cultivo, aumentou a porcentagem de lipídios totais dos rotíferos, atingindo uma média superior aos da Artemia sp., especialmente os derivados de W3, favorecendo positivamente o metabolismo das larvas e acarretando bom desempenho dos animais até a fase de pós-larva (Thomaz et al., 2002). Estes resultados corroboram resultados obtidos por vários autores, que afirmam aumento do valor nutritivo dos rotíferos após o enriquecimento

Tabela 1 - Sobrevivência das pós-larvas de $M$. rosenbergii submetidas a diferentes tratamentos alimentares, ao final do período experimental (35 dias)

Table 1 - Post-larvae survival of M. rosenbergii submitted to different feeding systems, at the end of the experimental period (35 days)

\begin{tabular}{lc}
\hline $\begin{array}{l}\text { Tratamentos } \\
\text { Treatments }\end{array}$ & $\begin{array}{c}\text { Sobrevivência (\%) } \\
\text { Survival (\%) }\end{array}$ \\
\hline T2 (100\% Artemia) & $68,36^{\mathrm{ns}}$ \\
T3 (60\% Artemia + 40\% Rotífero) & $68,76^{\mathrm{ns}}$ \\
T4 (40\% Artemia + 60\% Rotífero) & $64,60^{\mathrm{ns}}$ \\
\hline ns = não-significativo pelo teste qui-quadrado a $5 \%$ de probabi- \\
$\quad$ lidade.
\end{tabular}

R. Bras. Zootec., v.33, n.6, p.1928-1933, 2004 (Supl. 2)
(Hirayama \& Nakamura, 1976; Scott \& Baynes, 1978; Seixas Filho et al., 1985, 2000; Rainuzzo et al., 1989; Samocha et al., 1989; Kissil \& Koven, 1990; Carié et al., 1993; Barreto \& Cavalcanti, 1997; Galvão, 1988).

Portanto, os níveis de substituição parcial efetuados nos tratamentos T3 (60\% Artemia sp. + $40 \%$ B. plicatilis) e T4 (40\% Artemia sp. $+60 \%$ B. plicatilis), em relação à alimentação tradicional T2 (100\% Artemia sp.), indicam que, nas proporções utilizadas, mostraram-se eficientes para o desenvolvimento das larvas.

Os resultados obtidos ao final do experimento, em relação à média de peso seco de 80 pós-larvas de cada tratamento, foram 3,29; 3,08 e 3,38 mg, respectivamente, para os tratamentos T2, T3 e T4. Portanto, o efeito dos regimes alimentares testados, T2 (100\% Artemia sp.), T3 (60\% Artemia sp. $+40 \%$ B. plicatilis) e T4 (40\% Artemia sp. $+60 \%$ B. plicatilis) sobre o peso seco médio das pós-larvas, comprovou não haver diferença significativa $(\mathrm{P}>0,05)$ entre os tratamentos ao término do experimento (Tabela 2).

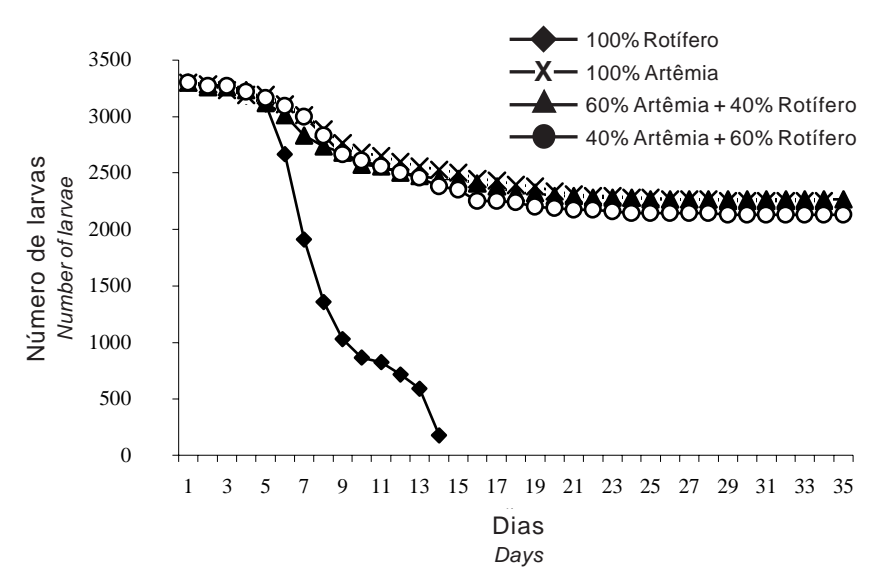

Figura 1 - Sobrevivência do número de larvas de $M$. rosenbergii nos tratamentos T1 (100\% B. plicatilis), T2 (100\% Artemia sp.), T3 (60\% Artemia sp. $+40 \%$ B. plicatilis) e T4 $(40 \%$ Artemia sp. $+60 \%$ B. plicatilis).

Figure 1 - Survival of the larvae number of M. rosenbergii in the treatments T1 (100\% B. plicatilis), T2 (100\% Artemia sp.), T3 (60\% Artemia sp. $+40 \%$ B. plicatilis) and T4 (40\% Artemia sp. $+60 \%$ B. plicatilis). 
Tabela 2 - Análise de variância do peso seco das pós-larvas, nos diferentes regimes alimentares testados

Table 2 - Analysis of variance of post-larvae dry weight tested at different feeding systems

\begin{tabular}{lcc}
\hline $\begin{array}{l}\text { Fonte de variação } \\
\text { Source of variation }\end{array}$ & $\begin{array}{c}\text { Graus de liberdade } \\
\text { Degrees of freedom }\end{array}$ & $\begin{array}{c}\text { Quadrado médio } \\
\text { Mean square }\end{array}$ \\
\hline $\begin{array}{l}\text { Tratamentos } \\
\text { Treatments } \\
\text { Blocos } \\
\begin{array}{l}\text { Blocks } \\
\text { Resíduo } \\
\text { Error }\end{array}\end{array}$ & 2 & $8,9775^{\text {ns }}$ \\
\hline
\end{tabular}

Coeficiente de variação (Coefficient of variation) $=16,36 \%$.

ns = não-significativo pelo teste Fisher, a $5 \%$ de probabilidade

$n s=$ not significant by Fisher test, at $5 \%$ of probability.

Este resultado se assemelha aos obtidos em larviculturas de camarão-d'água-doce submetidas a regimes alimentares tradicionais e naquelas onde houve substituição com o nematóide $P$. redivivus (Silva \& Rodrigues, 1997). Comparando-se os deste estudo (Tabelas 1 e 2), infere-se que a utilização parcial de rotíferos (B. plicatilis) enriquecidos e congelados na larvicultura do camarão-d'água-doce (M. rosenbergii) foi bem-sucedida.

Segundo Galvão et al. (1998), a utilização eficiente de B. plicatilis depende do sucesso da técnica de enriquecimento, que é capaz de afetar a composição bioquímica dos rotíferos (B. plicatilis), transformando-os em transportadores de vários produtos essenciais na alimentação de larvas de peixes e crustáceos (Barreto \& Cavalcanti, 1997).

Futuros estudos ainda são necessários para o aprimoramento das técnicas de substituição de náuplios de Artemia sp. por outros organismos vivos que proporcionem uma substituição parcial ou total dos náuplios de Artemia sp. e minimizem os custos de produção, a fim de viabilizar com maior autonomia a produção comercial da larvicultura do camarão-d'água-doce.

\section{Conclusões}

O rotífero B. plicatilis enriquecido e congelado não pode substituir totalmente os náuplios de Artemia sp. na larvicultura de $M$. rosenbergii.

A utilização do rotífero B. plicatilis enriquecido e congelado pode substituir parcialmente os náuplios de Artemia sp., no regime alimentar das larvas de $M$. rosenbergii, sem prejuízos na obtenção de póslarvas ou no crescimento e na sobrevivência de M. rosenbergii.

R. Bras. Zootec., v.33, n.6, p.1928-1933, 2004 (Supl. 2)

\section{Literatura Citada}

ALAM, M.J.; CHEAH, S.H.; ANG, K.J. Possible use of Moina spp. as a live feed substitute in larval rearing of the freshwater prawn, Macrobrachium rosenbergii (De Man). Aquaculture Fishery Management, v.22, n.4, p.531-535, 1991.

ALAM, M.J; ANG, K.J.; CHEAH, S.H. Use of Moina micrura (Kurz) as an Artemia substitute in the production of. Macrobrachium rosenbergii (De Man) post-larvae. Aquaculture, v.109, p.337-349, 1993a.

ALAM, M.J; ANG, K.J.; CHEAH, S.H. Weaning of Macrobrachium rosenbergii (De Man) larvae from Artemia to Moina micrura (Kurz). Aquaculture, v.12, p.187-194, 1993b.

ANIELLO, M. S.; SINGH, T. Some studies on the larviculture of the giant prawn (Macrobrachium rosenbergii). Elservier Scientific Publishing Company, v.10, p.225-232, 1980.

BARRETO, O.J.S.; CAVALCANTI, D.G. Enriquecimento de alimentos vivos para alimentação de larvas de organismos marinhos: uma breve revisão. Boletim do Instituto de Pesca, v.24, p.139-159, 1997.

BARROS, H.P.; VALENTI, W.C. Ingestion rates of Artemia nauolii for different larval stages of Macrobrachium rosenbergii. Aquaculture, v.217, n.(1-4), p.223-233, 2003.

CARIÉ, J.M.; NJRI, S.; SKARAMUCA, B. Dietary effects differents feeds on the biochemical composition of the rotifer (Brachionus plicatilis, Müller). Aquaculture, v.110, p.141-150, 1993.

CERQUEIRA, V.R. Sobrevivência, metamorfose e crescimento da larva do camarão rosa Penaeus paulensis, alimentado com o nematóide Panagrellus redivivus. [B.T.R.A.], v.3, n.3, 1989. p.13-10.

CESTAROLLI, M.A.; PORTELLA, M.C.; ROJAS, N.E.T. Efeitos do nível de alimentação e do tipo de alimento na sobrevivência e no desempenho inicial de larvas de curimbatá Prochilodus scrofa (STEINDACHNER, 1881). Boletim do Instituto de Pesca, v.24 (único), p.119-129, 1997.

CORREIA, E.S.; SUWANNATOUS, S.; NEW, M. Flow-through hatchery systems and management In: NEW, M.B.; VALENTI, W.C. (Eds.) Freshwater prawn culture: the farming of Macrobrachium rosenbergii. Oxford: Blackwell, 2000. p.52-68.

EUCLYDES, R.F. Sistema para análise estatísticas e genéticas. Manual de utilização do programa SAEG. Viçosa, MG: Universidade Federal de Viçosa, 1983. 59p. 
FREEMAN, J. Regulation of tissue growth in crustacean larvae by feeding regime. Bull. Mar. Biol. Lab. Woods Hole, v.178, n.3, p.217-221, 1990.

GALVÃO, M.S.N.; BARRETO, O.J.S.; YAMANAKA, N. Cultivo intensivo dos rotíferos Brachionus plicatilis $\mathbf{O}$. F. Muller, 1786 e Brachionus rotundiformis Tschugunoff, 1921 (Rotifera, Brachionidae). São Paulo: 1988. v.24, 15p.

HIRAYAMA, K.; NAKAMURA, K. Fundamental studies on the physiology of rotifers in mass culture. Aquaculture, v.8, p.301-307, 1976.

KISSIL, G.W.; KOVEN, W.M. Preparation of oils, enhanced in highly unsaturated fatty acid (HUFA) content, by low temperature crystallization separation, for rotifer (Brachiounus plicatilis) enrichment. Aquaculture, v.88, p.69-74, 1990.

LAVENS, P.; THONGROD, S.; SORGELOOS. P. Larval prawn feeds and the dietary importance of Artemia In: NEW, M.B.; VALENTI, W.C. (Eds.) Freshwater prawn culture: the farming of Macrobrachium rosenbergii. Oxford: Blackwell, 2000. p.91-111.

LOVETT, D.L.; FELDER, D.L. Evaluation of. the rotifer Brachionus plicatilis as a substitute for Artemia sp in feeding larvas of. Macrobrachium rosenbergii. Aquaculture, v.71, p.331-338, 1988.

NEW, M.B. A review of. dietary studies with shrimp and praws. Aquaculture, v.9, p.101-144, 1976.

NEW, M.B. Freshwater praw culture: a review. Aquaculture, v.88, p.99-143, 1990.

NEW, M.B. Status of freshwater prawn farming: a review. Aquaculture Research. v.26, n.1, p.1-54, 1995.

NEW, M.B.; D'ABRAMO, L.R.; VALENTI, W.C. et al. Sustainability of freshwater prawn culture. In: NEW, M.B.; VALENTI, W.C. (Eds.) Freshwater prawn culture: the farming of Macrobrachium rosenbergii. Oxford: Blackwell, 2000. p.429-443.

RAINUZZO, J.R.; OLSEN, Y.; ROSENLUND, G. The effect of enrichement diets on the rotifer Brachionus plicatilis. Aquaculture, v.79, p.157-161, 1989.

SAMOCHA, T.M.; UZIEL, N.; BROWDY, C.L. The effect of feeding two prey organisms, nauplii of Artemia and rotifers, Brachionus plicatilis upon suvival and growth of larval marine shrimp, Penaeus semisulcatus. Aquaculture, v.77, n.1, p.11-19, 1989.

SCOTT, A.P.; BAYNES, S.M. Effect of algal diet and temperature on the biochemical composition of the rotifers, Brachionus plicatilis. Aquaculture, v.14, n.3, p.247-260, 1978.

SEIXAS FILHO, J.T.; SIMÃO, O.M.; TRIANI, L. et al. Rotífero: uma alternativa no arraçoamento larval de Macrobrachium rosenbergii. [C.T.]: Pesagro, 1984. p. 1-3.

SEIXAS FILHO, J.T.; SOUZA, M.M.; CUNHA, L.L. et al. Ração artificial para larvas de Macrobrachium rosenbergii. [C.T.]: Pesagro, 1985, p.1-3.

SEIXAS FILHO, J.T.; TRIANI, L.; THOMAZ, L.A. et al. Utilização da morfometria na avaliação de larvas do camarão de água doce Macrobrachium rosenbergii (De Man, 1879) submetidas a diferentes regimes alimentares. In: SIMPÓSIO BRASILEIRO DE AQÜICULTURA-Simbraq, 11., 2000, Florianópolis. Anais... Santa Catarina: SIMBRAq, 2000. p.2-17.
SILVA, F.M.; RODRIGUES, J.B.R. Efeito da substituição de Artemia sp. pelo Nematóide Panagrellus redivivus sobre o crescimento e sobrevivência larval do camarão de água doce. Boletim do Instituto de Pesca, v.24 (especial), p.35-48, 1997.

SORGELOOS, P. The use of the brine shrimp Artemia in aquaculture In: PERSOONE, G.; SORGELOOS, P.; ROELS, O. et al. (Eds.) The brine shrimp Artemia. University Press: Wetteren, 1980. p.25-46.

SORGELOOS, P.; BOSSUYT, E.; LAVENS, P. et al. The use of brine shrimp Artemia in crustacean hatcheries and nurseries. In: Mc VEY, J.P. (Ed.) Handbook of maricult. Florida, 1983. v.1, p.71-96.

SORGELOOS, P.; LÉGER, P. Improved larviculture outputs of marine fish, shrimp and prawn. Journal World Aquaculture Society, v.23, n.4, p.251-264, 1992.

STOTTRUP, J.G.; ATTRAMADAL, Y. The influence of different rotifer and Artemia enrichment diets on growth, survival and pigmentation in turbot (Scophthlmus maximus L.) larvae. Journal World Aquaculture, v.3, n.4, p.307315, 1992.

STOTTRUP, J.G.; ATTRAMADAL, Y. The influence of different rotifer and Artemia enrichment diets on growth, survival and pigmentation in turbot (Scophthlmus maximus L.) larvae. Journal World Aquaculture, v.23, n.4, p.307-315, 1992.

THOMAZ, L.A.; OSHIRO, L.M.Y.; TRIANI, L. Avaliação Bromatológica de rotíferos, Brachionus plicatilis, (O. F. Muller, 1786) enriquecidos. In: CONGRESSO BRASILEIRO DE ZOOLOGIA, 24., 2001, Itajaí. Anais... Itajaí: Sociedade de Zoologia, 2002. p.271.

UNO, Y.; SOO, K.C. Larval development of. Macrobrachium rosenbergii reared in the laboratory. Journal Tokyo Fish, v.55, p.179-190, 1969.

VALENTI, W.C. Criação de camarão da Malásia. Jaboticabal: FUNEP, 1991. 53p.

VALENTI, W.C. Carcinicultura de água doce. Jaboticabal: FUNEP, 1998. 383p.

Van Der MEEREN, T. Selective feeding and prediction of food consumption in turbot larvae (Scophthalmus maximus L.) reared on the rotifer Brachionus plicatilis and natural zooplankton. Aquaculture, v.93, p.35-55, 1991.

WHYTE, J.C.N.; BOURNE, N.; HODGSON, C.A. Influence of algal diets on biochemical composition and energy reserves in Patinopecten yessoensis (lay) larvae. Aquaculture, v.78, p.333-347, 1989.

WHYTE, J.C.N.; CLARKE, W.C.; GINTHER, N.G. et al. Influence of composition of Brachionus plicatilis and Artemia on growth of larval sablefish (Anoplopoma fimbria, Pallas). Aquaculture, v.119, p.47-61, 1994.

YUFERA, M.; RODRIGUEZ, A.; LUBIAN, L.M. Zooplankton ingestion and feeding behavior of Penaeus kerathurus larvae reared in the laboratory. Aquaculture, v.42, p.217-224, 1985.

Recebido em: 07/11/02 Aceito em: 12/11/03 